\title{
Factores relacionados con la adherencia al sulfato ferroso en gestantes de los establecimientos de salud de la Micro Red Cono Sur de la Región de Salud de Tacna
}

\author{
Factors related to the adherence to ferrous sulfate in pregnant women of health \\ facilities of Micro Red Cono Sur of the Health Region of Tacna
}

\author{
Edith Rocío Godoy Gonzáles ${ }^{1 a}$ \\ ${ }^{1}$ Escuela Profesional de Obstetricia, Facultad de Ciencias de la Salud, Universidad Nacional Jorge Basadre Grohmann. \\ Tacna, Perú. \\ ${ }^{a}$ Licenciada en Obstetricia, Doctora en Ciencias de la Salud, Docente.
}

\section{Resumen}

Objetivo: Determinar factores relacionados con la adherencia al sulfato ferroso en gestantes de la Micro Red Cono Sur de la Región de Salud de Tacna.

Material y métodos: Estudio analítico transversal, en una muestra de 365 gestantes de cinco establecimientos del Ministerio de Salud del primer nivel de atención de la Micro Red Cono Sur del distrito Gregorio Albarracín Lanchipa de la provincia de Tacna, de julio a setiembre del 2017. Se aplicó un cuestionario. Resultados: Se encontró semejanzas en cuanto a los factores edad, paridad, olvido de la toma de tableta de sulfato ferroso, epigastralgia, diarrea, estreñimiento, náuseas, acidez, explicación específica de toma de sulfato ferroso, creencia que el sulfato ferroso es de riesgo para el feto $(\mathrm{p}>0,05)$ y predominio de adherencia inadecuada $(<75 \%)$ al sulfato ferroso. Basado en el análisis multivariado, se demostró que el factor olvido de toma de la tableta de sulfato ferroso $(\mathrm{p}=0,005)$; toma de otra medicación, además del sulfato ferroso $(\mathrm{p}=0.027)$ y creencia de que el sulfato ferroso constituye un riesgo para el feto $(\mathrm{p}=0,047)$; se relacionan significativamente con la adherencia al sulfato ferroso en las gestantes estudiadas. Conclusión: Los factores como olvido de toma de la tableta de sulfato ferroso, toma de otra medicación, además del sulfato ferroso, y creencia de que el sulfato ferroso constituye un riesgo para el feto, se relacionan significativamente con la adherencia al sulfato ferroso y tienen un poder de predicción suficiente para la adherencia inadecuada en las gestantes.

Palabras clave: Adherencia a medicamentos, sulfato ferroso, gestantes.

\begin{abstract}
Aim: To determine factors related to adherence to ferrous sulfate in pregnant women from the Micro Red Cono Sur of the Health Region of Tacna.

Material and methods: Cross-sectional analytical study, in a sample of 365 pregnant women from five health facilities of the Ministry of Health of the first level of care of the Micro Red Cono Sur of the Gregorio Albarracín Lanchipa district of Tacna, from July to September 2017, a questionnaire was applied.

Results. Similarities were found regarding the factors age, parity, forgetfulness of taking ferrous sulfate tablet, epigastralgia, diarrhea, constipation, nausea, acidity, specific explanation for taking ferrous sulfate, belief that ferrous sulfate is risk for the fetus $(\mathrm{p}>0.05)$ and predominance of inadequate adherence $(<75 \%)$ to ferrous sulfate. Based on the multivariate analysis, it was demonstrated that the forgetfulness factor of taking the ferrous sulfate tablet $(\mathrm{p}=0.005)$; taking other medication, in addition to ferrous sulfate $(\mathrm{p}=0.027)$ and belief that ferrous sulfate constitutes a risk for the fetus $(p=0.047)$ are significantly related to adherence to ferrous sulfate in pregnant women studied.

Conclusion: Factors such as forgetfulness of taking the ferrous sulfate tablet, taking other medication in addition to ferrous sulfate and belief that ferrous sulfate constitutes a risk to the fetus, are significantly related to adherence to ferrous sulfate and have sufficient prediction power for inadequate adherence in pregnant women.

Keywords: Medication adherence, ferrous sulfate, pregnant.
\end{abstract}




\section{Introducción}

La anemia se considera como el problema hematológico más frecuente en gestantes del mundo entero (1), y la deficiencia ferropénica como la principal causa de anemia en las gestantes, principalmente, debido a la demanda del feto, y a que muchas veces esa deficiencia no es cubierta por la dieta habitual.

El Ministerio de Salud del Perú, dentro del marco de la política sanitaria de lucha contra la anemia en gestantes, realiza la provisión gratuita de sulfato ferroso y ácido fólico; sin embargo, en muchos casos las gestantes no cumplen o abandonan el tratamiento, lo que determina una baja probabilidad de éxito de la prescripción. Esta situación se observa a menudo en los establecimientos de salud de la Micro Red Cono Sur de Tacna, lo que ha motivado la decisión de realizar el presente estudio, para aportar evidencias sobre los factores que podrían estar incidiendo en una adherencia inadecuada en la población de gestantes del ámbito geográfico de competencia de los establecimientos de salud C.S. San Francisco; C.S. Viñani; P.S. Vista Alegre; P.S. Las Begonias, y P.S. 5 de noviembre.

El objetivo fue determinar los factores que se relacionan con la adherencia al sulfato ferroso en gestantes de los establecimientos de salud de la Micro Red Cono Sur de la Región de Salud de Tacna.

\section{Material y métodos}

Se realizó un estudio analítico transversal, de julio a setiembre del 2017. La población de estudio fue de 1592 gestantes, y el tamaño de la muestra fue de 365 gestantes (20), atendidas en los establecimientos de salud de la Micro Red Cono Sur de la Región de Salud de Tacna, seleccionadas aleatoriamente según el registro de gestantes que obra en el Servicio de Obstetricia de los establecimientos de salud. Se incluyeron gestantes con prescripción de sulfato ferroso vía oral $>=60 \mathrm{mg} /$ día, a término, con feto único; previo consentimiento informado. Se excluyeron gestantes con problemas de discernimiento, que no les permita contestar el cuestionario, o con patologías crónicas (hipertensión arterial, diabetes mellitus, hemorragias o hemorroides).

Para realizar este trabajo se coordinó con la Escuela de Postgrado de la Universidad Católica de Santa María para la presentación del investigador, a fin de solicitar autorización a los Gerentes de cada establecimiento de salud. Para la recolección de datos se capacitó a dos entrevistadoras; se validó el instrumento mediante el Método Delphi. La técnica empleada fue la entrevista; se aplicó un cuestionario anónimo con el objetivo de medir seis factores (en 30 ítems) de administración individual, con una duración de aplicación de 15 minutos aproximadamente; y validado por expertos.

Para medir la variable adherencia al sulfato ferroso: se utilizó el formulario de preguntas de adherencia (6) con el objetivo de medir el nivel de adherencia al sulfato ferroso, de administración individual y una duración de aplicación de 5 a 7 minutos aproximadamente. Para el análisis estadístico se aplicó la prueba de ji al cuadrado en el análisis bivariado, y se realizó regresión logística.

Tabla 1. Adherencia al sulfato ferroso.

\begin{tabular}{lcc}
\hline Adherencia & $\mathbf{n}^{\circ}$ & $\mathbf{\%}$ \\
\hline Adherencia adecuada $(\geq 75 \%)$ & 154 & 42.2 \\
Adherencia inadecuada $(<75 \%)$ & 211 & 57.8 \\
Total & 365 & 100.0 \\
\hline
\end{tabular}

De las 365 gestantes participantes en el estudio se encontró que el $57.8 \%$ presentó adherencia inadecuada $(<75 \%)$. (Tabla 1).

Tabla 2. Factores sociodemográficos asociados con la adherencia al sulfato ferroso.

\begin{tabular}{|c|c|c|c|c|c|c|c|}
\hline & \multicolumn{2}{|c|}{$\begin{array}{c}\text { Adherencia } \\
\text { inadecuada }(< \\
75 \%)\end{array}$} & \multicolumn{2}{|c|}{$\begin{array}{c}\text { Adherencia } \\
\text { adecuada }(\geq \\
75 \%)\end{array}$} & \multicolumn{2}{|r|}{ Total } & \multirow[t]{2}{*}{$\begin{array}{c}\mathrm{X}^{2} \\
\text { p-valor }\end{array}$} \\
\hline & $\mathrm{N}^{\circ}$ & $\%$ & $\mathrm{~N}^{\circ}$ & $\%$ & $\mathrm{~N}^{\circ}=365$ & $\%=100$ & \\
\hline Edad & & & & & & & \\
\hline De 15 a 17 & 12 & 3.3 & 5 & 1.4 & 17 & 4.7 & $X^{2}=4.122$ \\
\hline De 18 a 29 & 127 & 34.8 & 108 & 29.6 & 235 & 64.4 & $\mathrm{p}$-valor $=0.127$ \\
\hline De 30 a 43 & 72 & 19.7 & 41 & 11.2 & 113 & 31.0 & \\
\hline \multicolumn{8}{|l|}{$\begin{array}{l}\text { Nivel } \\
\text { educativo }\end{array}$} \\
\hline Primaria & 14 & 3.8 & 6 & 1.0 & 20 & 5,5 & \\
\hline Secundaria & 144 & 39.5 & 105 & 28.8 & 249 & 68.2 & $X^{2}=2.316$ \\
\hline Superior & 52 & 14.2 & 43 & 11.8 & 95 & 26.0 & $\mathrm{p}$-valor $=0.509$ \\
\hline Analfabeta & 1 & 0.3 & 0 & 0.0 & 1 & 0.3 & \\
\hline \multicolumn{8}{|l|}{ Estado civil } \\
\hline Soltera & 29 & 7.9 & 19 & 5.2 & 48 & 13.2 & $X^{2}=0.179$ \\
\hline Casada & 24 & 6.6 & 17 & 4.7 & 41 & 11.2 & $\mathrm{p}$-valor $=0.915$ \\
\hline Conviviente & 158 & 43.3 & 118 & 32.3 & 276 & 75.6 & \\
\hline
\end{tabular}


En la Tabla 2 se muestra que el $64.4 \%$ de las gestantes tienen entre 18 a 29 años. Este porcentaje es mayor en las gestantes que presentan adherencia inadecuada (34.8\%), frente a las gestantes con adherencia adecuada (29.6\%). En cuanto al nivel educativo, la mayoría de las gestantes $(68.2 \%)$ presenta estudios secundarios; de las cuales, el $39.5 \%$ corresponde a gestantes con nivel secundaria y adherencia inadecuada, mientras que el $28.8 \%$ presentan adherencia adecuada y estudios secundarios. Por otro lado, prácticamente las tres cuartas partes son convivientes $(75.6 \%)$, de las cuales, la mayoría presentan adherencia inadecuada (43.3\%), y la diferencia de las convivientes presentan una adherencia adecuada al sulfato ferroso $(32.3 \%)$.

Tabla 3. Factores obstétricos asociados con la adherencia al sulfato ferroso.

\begin{tabular}{|c|c|c|c|c|c|c|c|}
\hline \multirow[b]{2}{*}{ Paridad } & \multicolumn{2}{|c|}{$\begin{array}{l}\text { Adherencia } \\
\text { inadecuada }(< \\
75 \%)\end{array}$} & \multicolumn{3}{|c|}{$\begin{array}{l}\text { Adherencia } \\
\text { adecuada }(\geq 75 \%)\end{array}$} & \multirow{2}{*}{$\begin{array}{c}\text { Total } \\
\%\end{array}$} & \multirow[t]{2}{*}{$\begin{array}{c}\mathrm{X}^{2} \\
\text { p-valor }\end{array}$} \\
\hline & $\mathrm{N}^{\circ}$ & $\%$ & $\mathrm{~N}^{\circ}$ & $\%$ & $\mathrm{~N}^{\circ}$ & & \\
\hline Nulípara & 90 & 24.7 & 64 & 17.5 & 154 & 42.2 & \multirow{3}{*}{$\begin{array}{c}\mathrm{X}^{2}=0.110 \\
\text { p-valor= } \\
0.947\end{array}$} \\
\hline Primípara & 64 & 17.5 & 46 & 12.6 & 110 & 30.1 & \\
\hline Multípara & 57 & 15.6 & 44 & 12.1 & 101 & 27.7 & \\
\hline \multicolumn{8}{|l|}{$\begin{array}{l}\text { Control } \\
\text { prenatal }\end{array}$} \\
\hline $\begin{array}{l}\text { Controlada (6 a } \\
+)\end{array}$ & 96 & 26.3 & 62 & 17.0 & 158 & 43.3 & $X^{2}=0.995$ \\
\hline $\begin{array}{l}\text { No controlada } \\
\text { (5) }\end{array}$ & 115 & 31.5 & 92 & 25.2 & 207 & 56.7 & $\begin{array}{c}\mathrm{p} \text {-valor }= \\
0.319\end{array}$ \\
\hline \multicolumn{8}{|l|}{$\begin{array}{l}\text { Preparación de } \\
\text { psicoprofilaxis } \\
\text { obstétrica }\end{array}$} \\
\hline $\begin{array}{l}\text { Con } \\
\text { preparación en } \\
\text { psicoprofilaxis }\end{array}$ & 8 & 2.2 & 8 & 2.2 & 16 & 4.4 & $X^{2}=0.1418$ \\
\hline $\begin{array}{l}\text { Sin preparación } \\
\text { en } \\
\text { psicoprofilaxis }\end{array}$ & 203 & 55.6 & 146 & 40.0 & 349 & 95.6 & $\begin{array}{c}\mathrm{p} \text {-valor }= \\
0.518\end{array}$ \\
\hline
\end{tabular}

En la Tabla 3, se observa que el $42.2 \%$ de las gestantes son nulíparas. Este porcentaje es mayor en las gestantes que presentan adherencia inadecuada (24.7\%), frente a las gestantes con adherencia adecuada (17.5\%). En cuanto al control prenatal, la mayoría de las gestantes $(56.7 \%)$ no es una gestante controlada; de las cuales, el $31.5 \%$ corresponde a gestantes con adherencia inadecuada. Por otro lado, casi las tres terceras partes no tienen preparación psicoprofiláctica (95.6\%), de las cuales, la mayoría, con un 55.6\%, presenta adherencia inadecuada (Tabla 3). No se encontró asociación en ninguno de los $\operatorname{casos}(\mathrm{p}>0.05)$.

Tabla 4. Factores dependientes de la terapéutica asociados con la adherencia al sulfato ferroso.

\begin{tabular}{|c|c|c|c|c|c|c|c|}
\hline & \multicolumn{2}{|c|}{$\begin{array}{l}\text { Adherencia } \\
\text { inadecuada } \\
(<75 \%)\end{array}$} & \multicolumn{2}{|c|}{$\begin{array}{r}\text { Adherencia } \\
\text { adecuada } \\
75 \%)\end{array}$} & \multicolumn{2}{|l|}{ Total } & $\begin{array}{c}\mathrm{X}^{2} \\
\text { p-valor }\end{array}$ \\
\hline $\begin{array}{l}\text { Dificultad para } \\
\text { ingerir la tableta }\end{array}$ & $\mathrm{N}^{\circ}$ & $\%$ & $\mathrm{~N}^{\circ}$ & $\%$ & $\begin{array}{l}N^{\circ}=3 \\
65\end{array}$ & $\begin{array}{c}\%=10 \\
0\end{array}$ & \\
\hline Sí & 88 & 24.1 & 43 & 11.8 & 131 & 35.9 & $X^{2}=7.351$ \\
\hline No & 123 & 33.7 & 111 & 30.4 & 234 & 64.1 & $\mathrm{p}$-valor $=0.007$ \\
\hline $\begin{array}{l}\text { Incomodidad por } \\
\text { tiempo de medicación } \\
\text { Sí } \\
\text { No }\end{array}$ & $\begin{array}{l}104 \\
107\end{array}$ & $\begin{array}{l}28.5 \\
29.3\end{array}$ & $\begin{array}{r}42 \\
112\end{array}$ & $\begin{array}{l}11.5 \\
30.7\end{array}$ & $\begin{array}{l}146 \\
219\end{array}$ & $\begin{array}{l}40.0 \\
60.0\end{array}$ & $\begin{array}{l}X^{2}=17.980 \\
p \text {-valor }=0.000\end{array}$ \\
\hline $\begin{array}{l}\text { Olvida tomar la tableta } \\
\text { Sí } \\
\text { No }\end{array}$ & $\begin{array}{r}182 \\
29\end{array}$ & $\begin{array}{r}49.9 \\
7.9\end{array}$ & $\begin{array}{r}104 \\
50\end{array}$ & $\begin{array}{l}28.5 \\
13.7\end{array}$ & $\begin{array}{r}286 \\
79\end{array}$ & $\begin{array}{l}78.4 \\
21.6\end{array}$ & $\begin{array}{l}X^{2}=18.402 \\
\text { p-valor }=0.000\end{array}$ \\
\hline $\begin{array}{l}\text { Toma además otra } \\
\text { medicación } \\
\text { Sí } \\
\text { No }\end{array}$ & $\begin{array}{r}66 \\
145\end{array}$ & $\begin{array}{l}18.1 \\
39.7\end{array}$ & $\begin{array}{r}28 \\
126\end{array}$ & $\begin{array}{r}7.7 \\
34.5\end{array}$ & $\begin{array}{r}94 \\
271\end{array}$ & $\begin{array}{l}25.8 \\
74.2\end{array}$ & $\begin{array}{l}X^{2}=7.987 \\
\text { p-valor }=0.005\end{array}$ \\
\hline
\end{tabular}


En la Tabla 4 se encuentra, que el $64.1 \%$ de las gestantes no tiene dificultad para ingerir la tableta de sulfato ferroso. Este porcentaje es mayor en las gestantes que presentan adherencia inadecuada (33.7\%), frente a las gestantes con adherencia adecuada (30.4\%). En cuanto a la incomodidad por el tiempo de medicación, la mayoría no presenta incomodidad (60\%); de las cuales, el $30.7 \%$ corresponde a gestantes sin incomodidad, pero con adherencia adecuada, mientras que el $29,3 \%$ presentan adherencia inadecuada y no incomodidad. Asimismo, el 78.4\%, se olvidan de tomar la prescripción de sulfato ferroso, de las que prácticamente el 50\% presenta adherencia inadecuada. En lo referente a tomar otra medicación; el 74.2\% lo niega, de las cuales, el 39.7\% presenta adherencia inadecuada; de igual modo, el 25.8\% sí toma otra medicación; de las cuales el 18.1\% también presenta adherencia inadecuada. En todos los casos la probabilidad es menor a 0.05 .

Se observa del análisis de regresión logística que, de los factores dependientes de la terapéutica se asocian a la incomodidad por tiempo de medicación, uso además de otra medicación y olvido de la toma con la adherencia al sulfato ferroso ( $\mathrm{p}<0.05)$.

Tabla 5. Factores efectos adversos de la terapéutica asociados con la adherencia al sulfato ferroso.

\begin{tabular}{|c|c|c|c|c|c|c|c|}
\hline \multirow[b]{3}{*}{ Epigastralgia } & \multicolumn{2}{|c|}{$\begin{array}{c}\text { Adherencia } \\
\text { inadecuada }(< \\
75 \%)\end{array}$} & \multicolumn{3}{|c|}{$\begin{array}{l}\text { Adherencia adecuada } \\
(\geq 75 \%)\end{array}$} & Total & \multirow[t]{2}{*}{$\begin{array}{c}\mathbf{X}^{2} \\
\text { p-valor }\end{array}$} \\
\hline & $\mathrm{N}^{\circ}$ & $\%$ & $\mathrm{~N}^{\circ}$ & $\%$ & $\mathrm{~N}^{\circ}=365$ & $\%=100$ & \\
\hline & & & & & & & \\
\hline Sí & 20 & 5.5 & 6 & 1.6 & 26 & 7.1 & $X^{2}=4.194$ \\
\hline No & 191 & 52.3 & 148 & 40.5 & 339 & 92.9 & $\mathrm{p}$-valor $=0.041$ \\
\hline \multicolumn{8}{|l|}{ Diarrea } \\
\hline Sí & 8 & 2.2 & 4 & 1.1 & 12 & 3.3 & $X^{2}=0.399$ \\
\hline No & 203 & 55.6 & 150 & 41.1 & 353 & 96.7 & $\mathrm{p}$-valor $=0.527$ \\
\hline \multicolumn{8}{|l|}{ Estreñimiento } \\
\hline Sí & 74 & 20.3 & 48 & 13.2 & 122 & 33.4 & $X^{2}=0.609$ \\
\hline No & 137 & 37.5 & 106 & 29.0 & 243 & 66.6 & $\mathrm{p}$-valor $=0.435$ \\
\hline \multicolumn{8}{|l|}{ Náuseas } \\
\hline Sí & 62 & 17.0 & 26 & 7.1 & 88 & 24.1 & $X^{2}=7.603$ \\
\hline No & 149 & 40.8 & 128 & 35.1 & 277 & 75.9 & $\mathrm{p}$-valor $=0.006$ \\
\hline \multicolumn{8}{|l|}{$\begin{array}{l}\text { Alteración } \\
\text { del sabor }\end{array}$} \\
\hline Sí & 50 & 13.7 & 28 & 7.7 & 78 & 21.4 & $X^{2}=1.611$ \\
\hline No & 161 & 44.1 & 126 & 34.5 & 287 & 78.6 & $\mathrm{p}$-valor $=0.204$ \\
\hline \multicolumn{8}{|l|}{$\begin{array}{l}\text { Intolerancia } \\
\text { al hierro }\end{array}$} \\
\hline Sí & 26 & 7.1 & 11 & 3.0 & 37 & 10.1 & $X^{2}=2.622$ \\
\hline No & 185 & 50.7 & 143 & 39.2 & 328 & 89.9 & $\mathrm{p}$-valor $=0.105$ \\
\hline
\end{tabular}

En la Tabla 5, respecto a la distribución de la adherencia inadecuada o adecuada y los efectos secundarios; se encontró que el 92.9\% niega epigastralgia; de las cuales el 52.3\% presenta adherencia inadecuada, frente a las gestantes con adherencia adecuada (40.5\%). El 96.7\% no presenta diarrea; de las cuales, el 55.6\% corresponde a gestantes con adherencia inadecuada, mientras que el $41.1 \%$ presenta adherencia adecuada. Las dos terceras partes de las gestantes (66.6\%) niegan presentar estreñimiento; de las cuales, el $37.5 \%$ presenta adherencia inadecuada. En lo referente a la presencia de náuseas, las tres cuartas partes (75.9\%) admite no presentar náuseas, de las que la mayoría, con un $40.8 \%$ presenta adherencia inadecuada, y la diferencia $35.1 \%$ no tiene náuseas y presenta adherencia adecuada. En cuanto a la alteración del sabor; más de las tres cuartas partes de las gestantes (78.6\%) niega este efecto; de las cuales, el $44.1 \%$ presenta adherencia inadecuada, y el $34.5 \%$ adherencia adecuada. Respecto a la intolerancia al hierro, la gran mayoría niega la molestia; empero, el 50.7\% no presenta intolerancia y su adherencia es inadecuada.

En el análisis de regresión logística, la somnolencia es un efecto adverso que se asocia con la adherencia al sulfato ferroso $(\mathrm{p}<0.05)$. 
Tabla 6. Factores efectos adversos de la terapéutica asociados con la adherencia al sulfato ferroso.

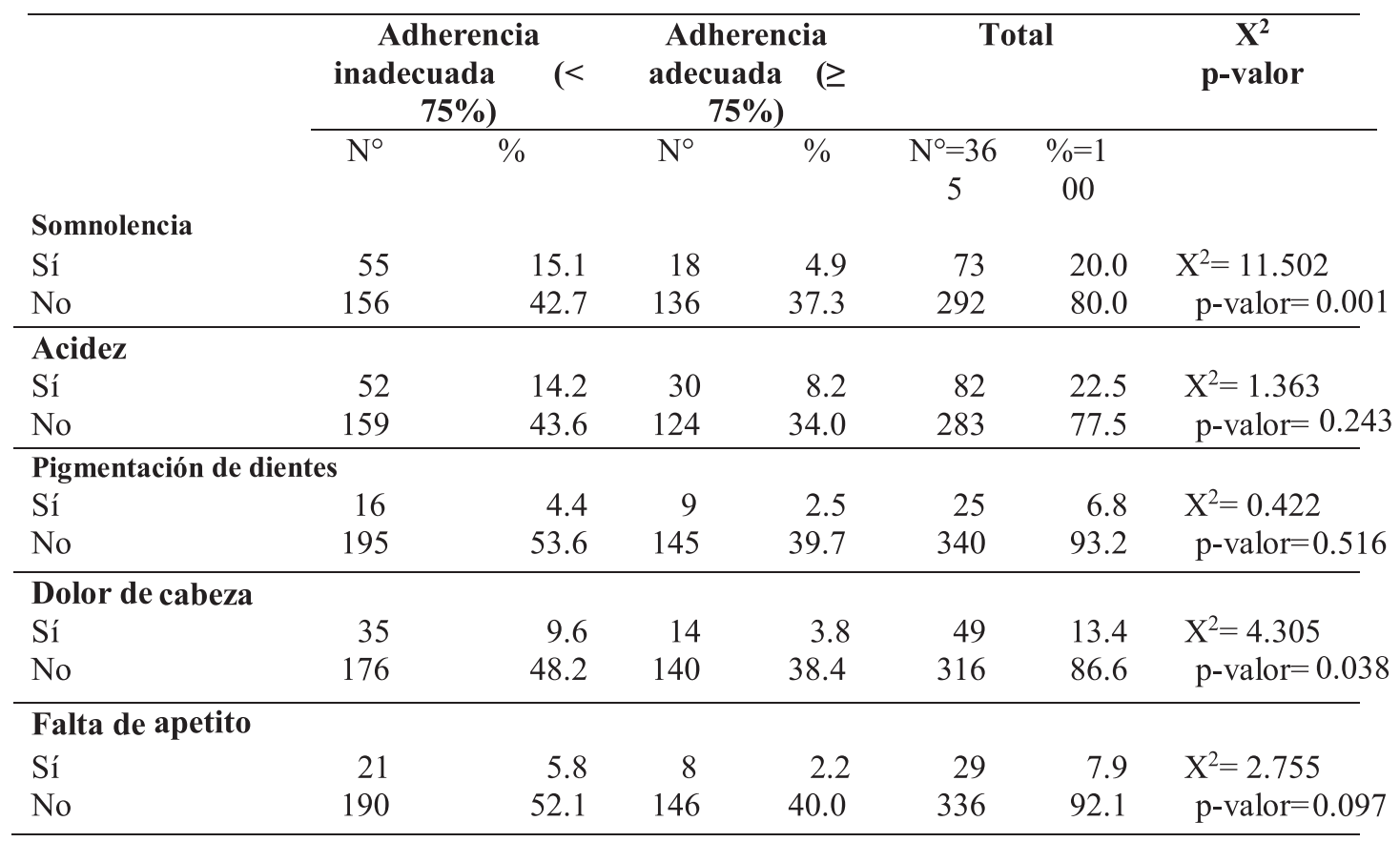

En la Tabla 6 se muestra, respecto a la distribución de la adherencia inadecuada o adecuada los efectos secundarios de la terapéutica, que el $80 \%$ niega somnolencia; de las cuales, el $42.7 \%$ presenta adherencia inadecuada, frente a las gestantes con adherencia adecuada $(37.3 \%)(\mathrm{p}<0.05)$. El 77.5\% no presenta acidez; de las cuales, el 43.6\% corresponde a gestantes con adherencia inadecuada, mientras que el 34\% presentan adherencia adecuada. Por otro lado, mayoritariamente las gestantes niegan presentar pigmentación de los dientes (93.2\%); de las cuales, el 53.6\% presenta adherencia inadecuada. En lo referente a la presencia de cefalea, el $86.6 \%$ refiere que no presenta cefalea; de las que, en su mayoría, con un $48.2 \%$ presenta adherencia inadecuada, y la diferencia $38.4 \%$ no presenta cefalea y su adherencia adecuada. $(\mathrm{p}<0.05)$. En cuanto a la inapetencia, se tiene que la gran mayoría niega la molestia (92.1\%); empero, el 52.1\% no presenta inapetencia y su adherencia es inadecuada.

Tabla 7. Factores relación facultativo - paciente asociados con la adherencia al sulfato ferroso.

\begin{tabular}{|c|c|c|c|c|c|c|c|}
\hline \multirow[b]{2}{*}{$\begin{array}{l}\text { Conformidad } \\
\text { con explicación de } \\
\text { razones de toma de SF }\end{array}$} & \multicolumn{2}{|c|}{$\begin{array}{c}\text { Adherencia } \\
\text { inadecuada } \\
(<75 \%) \\
\end{array}$} & \multicolumn{2}{|c|}{$\begin{array}{l}\text { Adherencia } \\
\text { adecuada } \\
(\geq 75 \%) \\
\end{array}$} & \multicolumn{2}{|c|}{ Total } & \multirow[t]{2}{*}{$\begin{array}{c}\mathbf{X}^{2} \\
\text { p-valor }\end{array}$} \\
\hline & $\mathrm{N}^{\circ}$ & $\%$ & $\mathrm{~N}^{\circ}$ & $\%$ & $\begin{array}{c}\mathrm{N}^{\circ}=36 \\
5\end{array}$ & $\%=100$ & \\
\hline Sí & 175 & 47.9 & 135 & 37.0 & 310 & 84.9 & $X^{2}=1.552$ \\
\hline No & 36 & 9.9 & 19 & 5.2 & 55 & 15.1 & $\mathrm{p}$-valor $=0.213$ \\
\hline \multicolumn{8}{|l|}{$\begin{array}{l}\text { Conformidad } \\
\text { de explicación } \\
\text { de efectos adversos }\end{array}$} \\
\hline $\begin{array}{l}\text { Sí } \\
\text { No }\end{array}$ & $\begin{array}{r}140 \\
71\end{array}$ & $\begin{array}{l}38.4 \\
19.5\end{array}$ & $\begin{array}{r}117 \\
37\end{array}$ & $\begin{array}{l}32.1 \\
10.1\end{array}$ & $\begin{array}{l}257 \\
108\end{array}$ & $\begin{array}{l}70.4 \\
29.6\end{array}$ & $\begin{array}{l}\mathrm{X}^{2}=3.957 \\
\mathrm{p} \text {-valor }=0.047\end{array}$ \\
\hline \multicolumn{8}{|c|}{ Confianza en facultativo } \\
\hline $\begin{array}{l}\text { Sí } \\
\text { No }\end{array}$ & $\begin{array}{r}173 \\
38\end{array}$ & $\begin{array}{l}47.4 \\
10.4\end{array}$ & $\begin{array}{r}140 \\
14\end{array}$ & $\begin{array}{r}38.4 \\
3.8\end{array}$ & $\begin{array}{r}313 \\
52\end{array}$ & $\begin{array}{l}85.8 \\
14.2\end{array}$ & $\begin{array}{l}X^{2}=5.796 \\
\text { p-valor }=0.016\end{array}$ \\
\hline \multicolumn{8}{|l|}{$\begin{array}{l}\text { Recibió consejería } \\
\text { específica } \\
\text { sobre sulfato ferroso }\end{array}$} \\
\hline $\begin{array}{l}\text { Sí } \\
\text { No }\end{array}$ & $\begin{array}{r}120 \\
91\end{array}$ & $\begin{array}{l}32.9 \\
24.9\end{array}$ & $\begin{array}{l}99 \\
55\end{array}$ & $\begin{array}{l}27.1 \\
15.1\end{array}$ & $\begin{array}{l}219 \\
146\end{array}$ & $\begin{array}{l}60.0 \\
40.0\end{array}$ & $\begin{array}{l}\mathrm{X}^{2}=2.039 \\
\mathrm{p} \text {-valor }=0.153\end{array}$ \\
\hline
\end{tabular}


En la Tabla 7 se muestra, respecto a la distribución de la adherencia inadecuada o adecuada y la relación facultativopaciente, que se encontró que cuando se les pregunta a las gestantes sobre la conformidad con la explicación que recibió sobre las razones por las que debía tomar sulfato ferroso, el $84.9 \%$ estuvo conforme; sin embargo, de este porcentaje la mayoría, con un $47.9 \%$ está conforme; pero su adherencia es inadecuada, frente a las que están conformes y presentan una adherencia adecuada (37\%).

Por otro lado, cuando se indagó sobre la conformidad de explicación de los efectos adversos; el 70.4\% admitió estar conforme; de las cuales, la mayoría con un 38.4 \% están conformes, pero su adherencia es inadecuada; en contraste, un $32,1 \%$ sí están conformes con la explicación sobre los posibles efectos adversos, y también presentan una adherencia adecuada ( $<0.05)$. En cuanto a la confianza que tienen en el facultativo que las atiende, mayoritariamente las gestantes admiten tener confianza (85.8\%); de las que la mayoría, con un 47.4\% manifiesta que sí tiene confianza, y su adherencia es inadecuada, frente a un $38.4 \%$ que tiene confianza en su facultativo y presenta una adherencia adecuada (p $<0.05)$. El $60 \%$ refiere que recibió una consejería específica sobre sulfato ferroso; de las que, en su mayoría, con un 32.9\%, presenta adherencia inadecuada, y la diferencia $(27.1 \%$ ) recibió consejería específica y su adherencia es adecuada.

Tabla 8. Factores creencias sobre el sulfato ferroso asociadas con la adherencia al sulfato ferroso.

\begin{tabular}{|c|c|c|c|c|c|c|c|}
\hline \multirow[b]{2}{*}{$\begin{array}{l}\text { Creencia de } \\
\text { que la } \\
\text { medicina } \\
\text { tradicional es } \\
\text { más efectiva }\end{array}$} & \multicolumn{2}{|c|}{$\begin{array}{l}\text { Adherencia } \\
\text { inadecuada }(< \\
75 \%)\end{array}$} & \multicolumn{3}{|c|}{$\begin{array}{l}\text { Adherencia } \\
\text { adecuada }(\geq 75 \%)\end{array}$} & Total & \multirow[t]{2}{*}{$\begin{array}{c}\mathbf{X}^{2} \\
\text { p-valor }\end{array}$} \\
\hline & $\mathrm{N}^{\circ}$ & $\%$ & $\mathrm{~N}^{\circ}$ & $\%$ & $\mathrm{~N}^{\circ}$ & $\%$ & \\
\hline Sí & 119 & 32.6 & 81 & 22.2 & 200 & 54.8 & $X^{2}=0.519$ \\
\hline No & 92 & 25.2 & 73 & 20.0 & 165 & 45.2 & $\mathrm{p}$-valor $=0.471$ \\
\hline \multicolumn{8}{|l|}{$\begin{array}{l}\text { Creencia de } \\
\text { que el sulfato } \\
\text { ferroso es } \\
\text { riesgoso para } \\
\text { el feto }\end{array}$} \\
\hline $\begin{array}{l}\text { Sí } \\
\text { No }\end{array}$ & $\begin{array}{r}42 \\
169\end{array}$ & $\begin{array}{l}11.5 \\
46.3\end{array}$ & $\begin{array}{r}13 \\
141\end{array}$ & $\begin{array}{r}3.6 \\
38.6\end{array}$ & $\begin{array}{r}55 \\
310\end{array}$ & $\begin{array}{l}15.1 \\
84.9\end{array}$ & $\begin{array}{c}X^{2}=9.142 \\
\text { p-valor }=0.002\end{array}$ \\
\hline \multicolumn{8}{|l|}{$\begin{array}{l}\text { Creencia de } \\
\text { que el sulfato } \\
\text { ferroso la } \\
\text { engorda }\end{array}$} \\
\hline Sí & 29 & 7.9 & 15 & 4.1 & 44 & 12.1 & $X^{2}=1.346$ \\
\hline No & 182 & 49.9 & 139 & 38.1 & 321 & 87.9 & $\mathrm{p}$-valor $=0.246$ \\
\hline
\end{tabular}

En la Tabla 8 se muestra que el $54.8 \%$ de las gestantes considera que la medicina tradicional es más efectiva. Este porcentaje es mayor en las gestantes que presentan adherencia inadecuada (32.6\%), frente a las gestantes con adherencia adecuada $(22.2 \%)$. En cuanto a la creencia de que el sulfato ferroso es riesgoso para el feto; del total, un $15.1 \%$ considera que el sulfato es riesgoso para el feto; de las cuales, el 11.5\% presenta adherencia inadecuada. Asimismo, del total, el 12.1\% considera que el sulfato ferroso la engorda; de las cuales, el 7.9\% presenta adherencia inadecuada. La prueba estadística de ji al cuadrado de asociación es significativa solo para la creencia de que el sulfato ferroso es riesgoso para el feto $(\mathrm{p}<0.05)$.

En el análisis de regresión logística sobre los factores dependientes de la terapéutica; se asocian a la incomodidad por tiempo de medicación; uso además de otra medicación, y olvido de la toma con la adherencia al sulfato ferroso $(\mathrm{p}<0.05)$. Asimismo, la somnolencia se asocia con la adherencia al sulfato ferroso $(\mathrm{p}<0.05)$. De igual manera, que de los factores creencias frente al medicamento; la creencia de que el sulfato ferroso es riesgoso para la salud del feto si se asocia con la adherencia al sulfato ferroso $(\mathrm{p}<0.05)$. 
Tabla 9. Regresión logística.

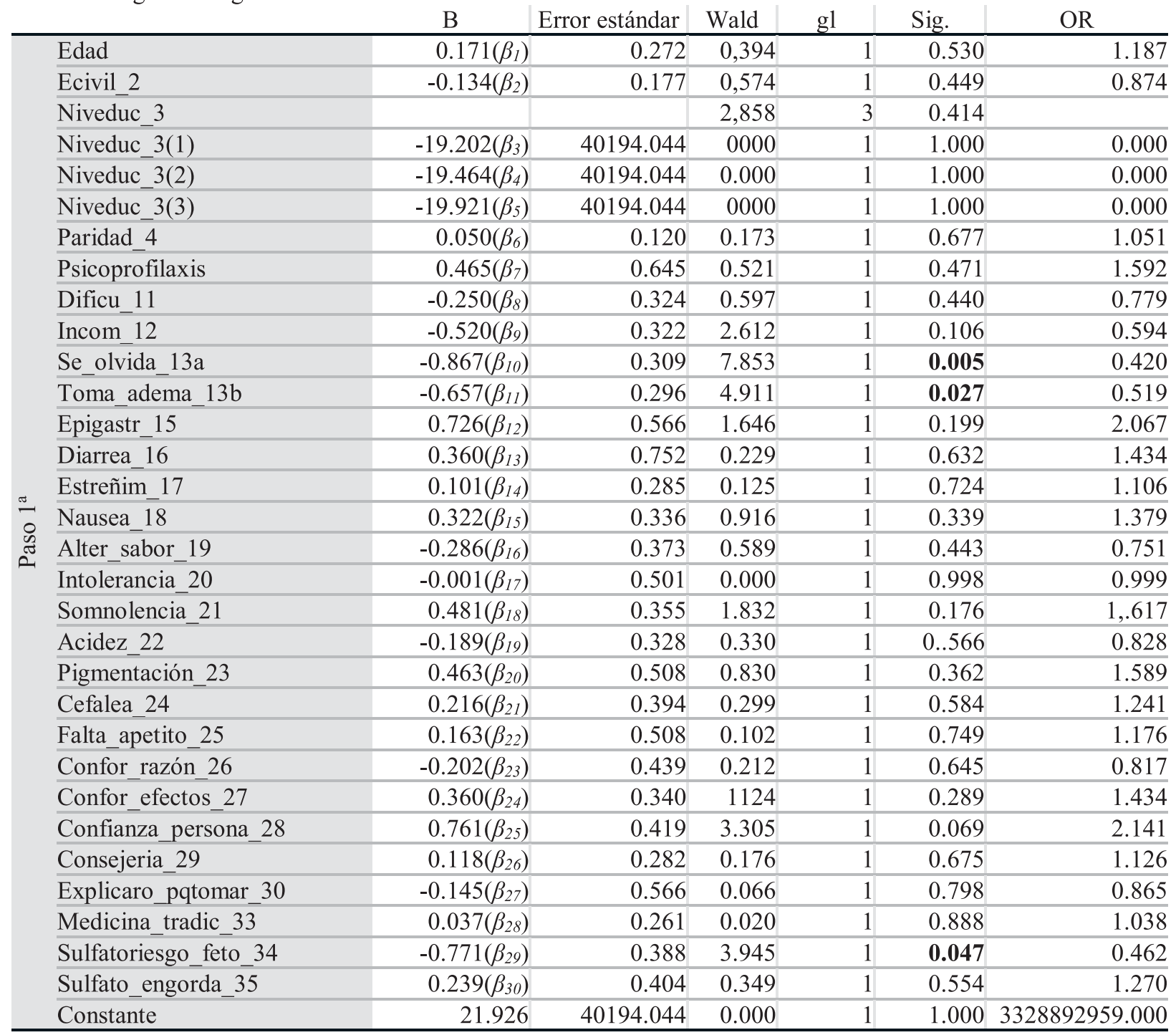

a. Variables especificadas en el paso 1: edad_cat, ecivil_2, niveduc_3, paridad_4, psico CAT, dificu_11, incom_12, se_olvida_13a, toma_adema_13b, epigastr_15, diarrea_16, estreñim_17, nausea_18, alter_sabor_19, intolerancia_20, somnolencia_21, acidez_22, pigmentacion_23, dolor_cabeza_24, falta_apetito_ 25 , confor_razon_26, confor_efectos_27, confianza_persona_28, no_consejeria_29, explicaron_pqtomar_30, medicina_tradic_33, sulfatoriesgo_feto_34, sulfato_engorda_ 35 .

En la Tabla 9 se muestra el análisis multivariado realizado con una regresión logística, para lo cual se ha utilizado una variable dependiente binaria dummy ( $1=$ Presencia y $2=$ Ausencia), siendo la intención pronosticar la vinculación entre las variables independientes (factores $=\mathrm{X}_{1}, \mathrm{X}_{2}, \mathrm{X}_{3} \ldots \mathrm{X}_{\mathrm{n}}$ ), y la variable dependiente ( $\mathrm{Y}=$ Adherencia al sulfato ferroso).

En tal sentido, se tiene los valores numéricos calculados para los coeficientes B del modelo, junto con la significancia (pvalor), bajo el presupuesto que cada coeficiente representa el cambio que se produce en el término Logit al elevarse en una "unidad la variable explicativa asociada" (20), factores creencias frente al medicamento. Los coeficientes $\boldsymbol{\beta}_{10}, \boldsymbol{\beta}_{11}, \boldsymbol{\beta}_{29}$, que corresponde a las variables se olvida de tomar la tableta de sulfato ferroso, toma además otra medicación además del sulfato ferroso, y la creencia de que el consumo de sulfato ferroso constituye un riesgo para el feto presentan p-valores menores que $0.05(0.005 ; 0.027 ; 0.047$ respectivamente).

Los resultados evidencian un comportamiento diferente de las variables en los establecimientos estudiados y en general, una baja adherencia al sulfato ferroso. 


\section{Discusión}

En el presente estudio, se encontró que un factor correspondiente a la dimensión factores dependientes de la terapéutica como el olvido de la toma de la prescripción de sulfato ferroso, tomar otra medicación, además, la creencia de que el sulfato ferroso es riesgoso para el bienestar del feto, se relacionan con la adherencia al sulfato ferroso $(\mathrm{p}<0.05)$. Asimismo, el $57.8 \%$ de las gestantes, en general de los cinco establecimientos de salud del primer nivel estudiados, presentan una adherencia inadecuada a la suplementación de hierro; lo cual, sin duda, disminuye la posibilidad de asegurar la eficacia de la administración de hierro durante el embarazo, como medida de salud pública, a favor de reducir el riesgo de la anemia en la madre, bajo peso neonatal, entre otros.

La adherencia adecuada a la suplementación de hierro en las gestantes estudiadas no supera el $57.4 \%$ si se compara estas proporciones en los diferentes establecimientos de salud, cifra que corresponde al P.S. Las Begonias. Por el contrario, el P.S. Vista Alegre es el establecimiento con el porcentaje más alto en adherencia inadecuada con un $67.9 \%$, lo que sugiere la necesidad de formular estrategias comunicativas para elevar la proporción de gestantes que toman correctamente y no descontinúan la prescripción del sulfato ferroso.

Del análisis bivariado, ninguna variable sociodemográfica se relaciona estadísticamente con la adherencia al sulfato ferroso $(p>0.05)$ en los cinco establecimientos; sin embargo, la tendencia de las proporciones más altas de la edad, el nivel educativo y el estado civil se concentran en la categoría de adherencia inadecuada, lo que sugiere que las mujeres jóvenes entre 18 y 29 años, con nivel de educación secundaria y las convivientes pueden tener una baja adherencia al sulfato ferroso, resultados concordantes con la afirmación de Merino (15), en cuanto al nivel educativo, ya que este considera que el nivel educativo está vinculado con la comprensión cabal de las indicaciones, reflexión y raciocinio de las bondades de la suplementación con sulfato ferroso.

Asimismo, para la dimensión factores dependientes de la terapéutica, el análisis bivariado reveló que en general la mayoría no presentó dificultad para ingerir la tableta, tampoco incomodidad con relación al tiempo que duraba la toma o prescripción para tomar el sulfato ferroso; sin embargo, la mayoría asintió que en muchas ocasiones se olvidaban de tomar la prescripción y que, además, no tomaba otra medicación, variables que se asociaron a la adherencia al sulfato ferroso en las gestantes estudiadas. Igualmente, en los efectos adversos de la terapéutica de sulfato ferroso en gestantes, se halló una relación significativa entre efectos adversos como epigastralgia y presencia de náuseas, resultados coincidentes con los de Merino, Lozano y Torrico (15); quienes reportaron que la aparición de náuseas durante el periodo de suplementación influye en una adherencia inadecuada. Igualmente, con los hallazgos de Anglas (14), quien reporta en un estudio empírico que las náuseas y vómitos se relacionan con la adherencia al sulfato ferroso en una muestra de gestantes peruanas; de igual forma con los resultados de Chapi (7) en el Perú, quien verificó una asociación entre la presentación de náuseas y la adherencia. También, existe coincidencia con las afirmaciones de Munayco, Arias, Gambirazio y Suárez (16), quienes afirman que el riesgo de presentar una adherencia inadecuada se asocia con la frecuencia de efectos adversos propios del suplemento de sulfato ferroso, al igual que con Huamán (17), quien también reportó que las náuseas y vómitos tienen una asociación con el incumplimiento del tratamiento de sulfato ferroso. Igualmente, algunos efectos adversos atribuidos por las gestantes a la suplementación de sulfato ferroso, y que se asocian con la adherencia, son la somnolencia y cefalea, que se vinculan estadísticamente con la adherencia inadecuada al sulfato ferroso en las gestantes que participaron en el estudio. Resultados similares halló Huamán (17) en una muestra de 80 casos, en donde el dolor de cabeza sí se asocia estadísticamente con el cumplimiento de la ingesta del sulfato ferroso en gestantes de 15 a 35 años.

Con respecto, a la relación facultativo-paciente, en tanto se afirma que "es un hecho que la participación del paciente en la toma de decisiones terapéuticas mejora la eficiencia terapéutica" (17); en este sentido, hallamos que la variable conformidad con la explicación que le dio el facultativo sobre los efectos adversos y la confianza con su tratante, presentaron una asociación estadística con la adherencia al tratamiento; sin embargo, las gestantes a pesar de tener la confianza y estar conformes con las explicaciones, presentan un mayor porcentaje de adherencia inadecuada; lo cual sugeriría que el contenido o la comunicación del mismo no es suficiente o efectiva para lograr un convencimiento real por parte de la paciente dentro de un esquema de manejo integral de la gestante.

El significado de los hallazgos permite resaltar que los factores sociodemográficos, nos referimos a la edad, estado civil al igual que en otros estudios (17), mayormente no se asocian con la adherencia al sulfato ferroso, más aún en el caso de la edad, son las gestantes de 18 a 43 años las que mayormente presentan adherencia inadecuada (aunque estadísticamente no están asociadas), lo que nos lleva a sugerir la inclusión de una variable de control como la situación laboral (si trabaja o no), ya que es posible que la implicancia laboral, la distraiga de la toma, o que considere que algún efecto secundario limita su rendimiento laboral. Otro aspecto importante, es que los resultados revelan que las gestantes tienen confianza en su facultativo; es decir, se aprecia una relación facultativo-paciente, positiva; lo que teóricamente debería traducirse en una conducta coincidente con el consejo médico (18), lo cual no se aprecia en los hallazgos 
que hemos obtenido, ya que la mayoría de las gestantes presenta una adherencia inadecuada. Lo que, a nuestro criterio, posibilita implicar en estudios futuros la cantidad y la calidad de la información que se le brinde a la gestante, de tal forma, que sea posible incidir en los aspectos que subyacen en la manifestación conductual de los seres humanos.

De igual forma la creencia de que la toma de sulfato ferroso puede dañar al feto incide en la adherencia, y revela una motivación y conducta emocional, o sentimientos de protección del hijo, situación fáctica que requiere procurar desmitificar creencias erróneas basadas en evidencia empírica.

Se concluye que los factores como olvido de toma de la tableta de sulfato ferroso $(\mathrm{p}=0.005)$; toma de otra medicación, además del sulfato ferroso $(\mathrm{p}=0.027)$ y creencia de que el sulfato ferroso constituye un riesgo para el feto $(p=0.047)$, se relacionan significativamente con la adherencia al sulfato ferroso y tienen un poder de predicción suficiente para la adherencia inadecuada en las gestantes estudiadas. Los factores socio-demográficos como la edad, nivel educativo y estado civil no se relacionan con la adherencia al sulfato ferroso en gestantes $(p>0.05)$. Los factores obstétricos como paridad, número de controles prenatales y número de sesiones de psicoprofilaxis no se relacionan con la adherencia al sulfato ferroso en gestantes $(p>0.05)$. Los factores dependientes terapéuticos como incomodidad por tiempo de medicación, uso, además, de otra medicación, olvido de toma de sulfato ferroso, se relacionan con la adherencia al sulfato ferroso en gestantes $(p<0.05)$. Los factores efectos adversos como; epigastralgia, diarrea, estreñimiento, náuseas, alteración del sabor, intolerancia al hierro, acidez, pigmentación de dientes, cefalea y falta de apetito no se relacionan con la adherencia al sulfato ferroso en gestantes ( $p>0-05)$, más sí se relaciona el factor somnolencia $(p<0.05)$. El factor facultativo-paciente, en términos de conformidad con explicación de razones para la toma de sulfato ferroso; explicación de efectos adversos, confianza en el facultativo y consejería de suplementación, no se relacionan con la adherencia al sulfato ferroso en gestantes $(p>0.05)$. Respecto a los factores creencias frente al medicamento, se halló que la creencia que el sulfato ferroso es riesgoso para el feto se relaciona significativamente con la adherencia al sulfato ferroso $(\mathrm{p}<0.05)$, no así las creencias de que la medicina tradicional es más efectiva o que incrementa el peso de la madre $(\mathrm{p}>0.05)$.

\section{Referencias bibliográficas}

1. Marín G, Fazioa P, Rubboa S, Baistrocchia A, Sagera G, Gelemur A. Prevalencia de anemia del embarazo y análisis de sus factores condicionantes. Publicaciones de medicina y ciencias de la salud - ELSEVIER [Revista en internet]. 2002 [Consultado 08 abril 2017]; $29(3)$ : $158-163$. D is p o n i b le en:http://www.elsevier.es/es-revista-atencionprimaria-27-articulo-prevalencia-anemia-delembarazo-analisis-13026984

2. Munares O, Gómez G. Niveles de hemoglobina $\mathrm{y}$ anemia en gestantes adolescentes atendidas en establecimientos del Ministerio de salud del Perú, 2009-2012. Revista peruana de medicina experimental y salud publica [Revista en internet] 2014. [Consultado 24 junio 2017]; D i s p o n i b 1 e e $\mathrm{n}$ : https://www.scielosp.org/scielo.php?pid=S1726 $-46342014000300014 \&$ script $=$ sci_arttext\&tlng $=\mathrm{pt}$

3. Moreno A. Prevalencia de anemia en mujeres embarazadas que acuden a la consulta en el servicio de urgencias del 1 de enero a diciembre del 2011 en el Hospital de ginecología y obstetricia del IMIEM [Tesis]. México:
Universidad Autónoma del Estado de México; $2005 \quad$ D i s p o n i b l e en:http:ri.uaemex.mx/bitstream/handle//396520 .pdf? sequence $=1$

4. Fernández M. Importancia de la administración de sulfato ferroso en el embarazo [Internet]. 2012 [Consultado 22 junio 2017]; Disponible en: http://embarazohierro.blogspot.pe/2012/05/imp ortancia-de-la-administracion-de.html

5. Ministerio de Salud del Perú. Plan nacional de reducción y control de la anemia en la población materno infantil en el Perú: 2017 - 2021. Lima; 2017 [Consultado 02 diciembre 2017]; $\begin{array}{lllllllllll}\mathrm{D} & \mathrm{i} & \mathrm{s} & \mathrm{p} & \mathrm{o} & \mathrm{n} & \mathrm{i} & \mathrm{b} & 1 & \mathrm{e}\end{array}$ en:http:/www.minsa.gob.pe/portada/Especiales /2016/anemia/documentos/1\%20Plan\%20Anem ia.pdf

6. Ministerio de Salud del Perú. Resolución Ministerial $N^{\circ}$ 069-2016. Directiva sanitaria para la prevención y control de la anemia por deficiencia de hierro en gestantes y puérperas. Lima; 2016.

7. Chapi G. Adherencia y factores asociados a la suplementación de hierro en gestantes anémicas en el Hospital Santa Rosa, Pueblo Libre [Tesis]. 
Perú: Universidad Nacional Mayor de San Marcos de Lima; 2014.

8. Organización Mundial de la Salud. Adherencia a los tratamientos a largo plazo. Pruebas para la acción [Traducción]. Organización Panamericana de la Salud; 2004. [Consultado 19 m a y o 20017 ]; D is p o n ible e n : http://www.amro.who.int/Spanish/AD/DPC/NC /nc-adherencia.pdf

9. López L, Romero S, Parra D, Rojas L. Adherencia al tratamiento: Concepto y medición. Hacia promoción de la salud [Revista en internet]. 2015 [Consultado 07 diciembre 2016]; 21(1): $117-137$. Disponible en: http://www.scielo.org.co/pdf/hpsal/v21n1/v21n 1a10.pdf

10. Rodríguez M, García E, Amariles P, Rodríguez A, Faus M. Revisión de test de medición del cumplimiento terapéutico utilizado en la práctica clínica. Publicaciones de Medicina y Ciencias de la Salud - ELSEVIER [Revista en internet]. 2008 [Consultado 05 abril 2017]; 40(8): 413-417. Disponible en: http://www.elsevier.es/es-revistaatencion-primaria-27-articulo-revision-testsmedicion-del-cumplimiento-13125407

11. San Gil C, Villazán C, Ortega Y. Caracterización de la anemia durante el embarazo y algunos factores de riesgo asociados, en gestantes del municipio. Revista cubana medicina general integral [Revista en internet]. 2014 [Consultado 02 junio 2017]; 30(1): 71-81. Disponible en: h t t p ://www.medigraphic.com/cgi$\mathrm{bin} / \mathrm{new} /$ resumen.cgi?idarticulo $=51344-2014$

12. Botella J, Clavero J. Tratado de Ginecología: Fisiología, obstetricia, perinatología, ginecología, reproducción. Madrid: Edit. Díaz de Santos, 1993.

13. Bothwell T. Iron requirements in pregnancy and strategies to meet them. The American Journal of clinical nutrition J Clin Nutr. 2000; 72(1), p. 257 264.
14. Anglas V. Adherencia y factores que influyen en la suplementación con hierro en gestantes que acuden al centro materno infantil Manuel Barreto, durante los meses de junio a agosto del 2015 [Tesis]. Perú: Universidad Nacional Mayor de San Marcos, 2015.

15. Merino V, Lozano D, Torrico F. Factores que influyen en la adherencia a la suplementación con sulfato ferroso durante el embarazo. Gaceta Médica Boliviana [Revista en internet]. 2010 [Consultado 06 julio 2017]; 33(2): 293-312. D i s p o n i b l e e n : http://www.scielo.org.bo/scielo.php?script=sci arttext\&pid $=$ S1012-29662010000200006

16. Munayco C, Arias L, Gambirazio C, Suárez L. Estudio de adherencia a la suplementación con hierro durante la gestación en las Direcciones de Salud de Apurímac y Ayacucho. Lima: Dirección General de Epidemiología del Ministerio de Salud. 2009.

17. Huamán J. Factores asociados al incumplimiento de la ingesta de sulfato ferroso en gestantes de 15 a 25 años [Tesis]. Perú: Universidad Privada Antenor Orrego, 2017 D i s p o n i b l e e n : http://repositorio.upao.edu.pe/bitstream/upaore p/3545/1/re maest_obst jessica.huam $\%$ c 3\%81 $\mathrm{n}$ _incumplimeinto. $\overline{d e}$ e.la.ingesta_datos.pdf

18. Celedón C. Relación médico paciente. Revista otorrinolaringología y cirugía de cabeza y cuello [Revista en internet]. 2016 [Consultado 01 agosto 2017]; 76(1): 51-54. Disponible en: http://www.scielo.org.bo/scielo.php?script=sci arttext\&pid $=$ S1012-

19. Murray, R. y Larry, S. Estadística. México, Editorial McGraw Hill, 2009.

20. Abanto, W. Diseño y desarrollo del proyecto de investigación. Guía y aprendizaje. Trujillo: Universidad Cesar Vallejo, 2015.
Correspondencia:

edithgodoy2@hotmail.com
Fecha de recepción: 16 de marzo del 2020

Fecha de aceptación: 25 de mayo del 2020 